ARTIGO ORIGINAL

\title{
Conteúdo relativo de água afeta o comportamento espectral de folhas de Eucalyptus spp.
}

\author{
Influence of relative water content variation on the spectral behavior of \\ Eucalyptus spp.

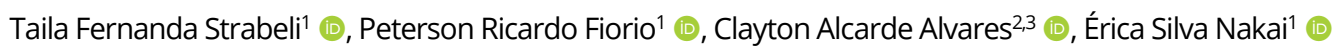

${ }^{1}$ Escola Superior de Agricultura "Luiz de Queiroz" - ESALQ, Universidade de São Paulo - USP, Piracicaba, SP, Brasil ${ }^{2}$ Faculdade de Ciências Agronômicas - FCA, Universidade Estadual Paulista "Júlio de Mesquita Filho" - UNESP, Botucatu, SP, Brasil ${ }^{3}$ Suzano SA., Limeira, SP, Brasil
\end{abstract}

Como citar: Strabeli, T. F., Fiorio, P. R., Alvares, C. A., \& Nakai, E. S. (2020). Conteúdo relativo de água afeta o comportamento espectral de folhas de Eucalyptus spp. Scientia Forestalis, 48(128), e3326. https://doi.org/10.18671/scifor.v48n128.25

\begin{abstract}
Resumo
A disponibilidade hídrica é fundamental para o desenvolvimento da planta e sua escassez limita o aumento de incremento florestal, provocando respostas específicas em cada genótipo de Eucalyptus spp.. Neste estudo, mediu-se a influência da variação do conteúdo relativo de água (CRA) no comportamento espectral foliar, pelo método da desidratação, em 11 genótipos de Eucalyptus spp.. As folhas amostradas foram secas progressivamente e, após a pesagem, foi realizada uma leitura em câmera hiperespectral. A água tem maior domínio de reflectância no infravermelho médio (1300 nm a 2500 nm). As regiões próximas a 1400 nm e 1900 nm apresentaram maior sensibilidade à variação do CRA. Uma classificação dos dados espectrais das folhas com maior e menor CRA foi feita com análise de componentes principais (ACP). Após essa análise, os comprimentos de onda $1450 \mathrm{~nm}, 1887 \mathrm{~nm}$ e $2101 \mathrm{~nm}$ foram os mais eficientes em classificar o progresso do nível da desidratação, que foi confirmado pelo teste de Tukey. Portanto, a variação do CRA foi inversa à resposta espectral em folha de Eucalyptus spp., principalmente na região do infravermelho médio. Assim, quanto menor a quantidade de água na folha, maior o fator de reflectância. Entre os diferentes genótipos estudados, o E. benthamii apresentou um menor fator de reflectância no infravermelho próximo e médio.
\end{abstract}

Palavras-chave: Sensibilidade ao conteúdo de água; Diferença de reflectância; Análise de componentes principais; Hiperespectral.

\begin{abstract}
Water availability is fundamental for the development of the plant and its scarcity limits forest growth, triggering specific responses in each species of Eucalyptus spp.. In this study the influence of the relative water content (CRA) through dehydration on the spectral behavior, was measured on leaves of 11 genotypes of Eucalyptus spp.. Sampled leaves were dried progressively and, after weighing, a hyperspectral reading was made. Water has a higher reflectance in the medium infrared (1300 nm at $2500 \mathrm{~nm}$ ). The greatest variation occurred between the wavelengths of $1300 \mathrm{~nm}-2500 \mathrm{~nm}$. The regions near $1400 \mathrm{~nm}$ and $1900 \mathrm{~nm}$ showed greater sensitivity to water content variation. A classification of the leaves' spectral data with major and minor CRA was done with Principal Components Analysis (PCA). After this analysis, the wavelengths $1450 \mathrm{~nm}, 1887 \mathrm{~nm}$ and $2101 \mathrm{~nm}$ were the most efficient in classifying the progress level of dehydration, which was confirmed by the Tukey test. Therefore, the CRA variation was inversely related to the spectral response of Eucalyptus spp., mostly in the medium infrared region. Thus, the smaller the amount of water in leaf, the higher the reflectance factor. Among the different genotypes studied $E$. benthamii resulted in a lower reflectance factor in the near and medium infrared.
\end{abstract}

Keywords: Sensibility to water content; Reflectance difference; Principal components analysis; Hyperspectral.

Fonte de financiamento: FAPESP Proc. n. 2013/22435-9.

Conflito de interesse: Nada a declarar.

Autor correspondente: ericanakai@usp.br

Recebido: 4 abril 2019.

Aceito: 18 novembro 2019

Editor: Paulo Henrique Müller Silva.

(c) Este é um artigo publicado em acesso aberto (Open Access) sob a licença Creative Commons Attribution, que permite uso, distribuição e reprodução em qualquer meio, sem restrições desde que o trabalho original seja corretamente citado. 


\section{INTRODUÇÃO}

O gênero Eucalyptus spp. tem destaque no setor florestal brasileiro, devido aos seus diversos usos, rápido crescimento, e ampla adaptação aos climas e solos do país (Flores et al., 2018). Devido a sua importância no setor florestal, é essencial entender a fisiologia dessa espécie, principalmente sua relação com a água, pois a disponibilidade hídrica é um dos principais limitadores da produção florestal (Campoe et al., 2016; Elli et al., 2019).

O conteúdo relativo de água (CRA), dado em porcentagem (\%), é uma medida universal utilizada para estimar a quantidade de água na folha (Datt, 1999; Sun et al., 2014; Ranjan et al., 2015). Permite determinar a tolerância das plantas à seca e sua adaptação a ambientes favoráveis (Arndt et al., 2015), pois apresenta forte relação com o volume celular (Jones, 1992).

O cálculo do CRA da vegetação tem sido obtido por diferentes métodos, como o tradicional de laboratório que envolve a pesagem seguida de submersão da folha em água e a secagem. Contudo, existem problemas metodológicos na determinação da massa úmida por interferência da respiração e fotossíntese (Dutra et al., 2011) e massa túrgida (Arndt et al., 2015) pelo ganho ou perda de massa dos tecidos. Esses problemas dificultam a sensibilidade do CRA e sua aplicação na folha (Dutra et al., 2011).

A radiometria de laboratório para determinação de deficiência hídrica apresenta bons resultados, de forma ágil e eficiente, através de modelos matemáticos (Sun et al., 2014; Cao et al., 2015). Gao (1996) afirmou que o comportamento espectral é sensível a quantidade de água na folha, porém foi analisado apenas a variação espectral das folhas, secas e úmidas, e os conteúdos de água intermediários não foram considerados.

A absorção de radiação eletromagnética pela água, em folhas, tende a diminuir a reflectância. Quando a água é perdida, devido aos processos fisiológicos da planta, há um aumento da reflectância mais expressivo entre os comprimentos de onda $(\lambda)$ de $1300 \mathrm{~nm}$ a $2500 \mathrm{~nm}$ no fator de reflectância (Carter, 1991), porém a reflectância também se altera entre $400 \mathrm{~nm}$ e $1300 \mathrm{~nm}$.

Na faixa do visível (400 - $760 \mathrm{~nm}$ ), ocorre baixa reflectância na folha devido a absorção da radiação eletromagnética pelos pigmentos (clorofila, xantofila e caroteno) dos cloroplastos. $\mathrm{Na}$ faixa espectral de 760 a $1300 \mathrm{~nm}$, as paredes celulares influenciam a reflectância, quanto mais lacunas entre células paliçádicas e mesófilo esponjoso, maior é o espalhamento interno da radiação incidente. A reflectância de 760 a $1300 \mathrm{~nm}$ é alta para impedir o aumento da temperatura foliar e evitar danos nas proteínas e açúcares, revelando um processo controlado pela morfologia e estrutura interna das folhas. De 1300 a 2500 nm, a reflectância depende do tamanho da célula, onde células maiores têm menores mudanças de direção da luz (Meneses et al., 2019).

A avaliação do estado hídrico das plantas é um dos fatores necessários para o monitoramento de culturas. Em culturas agrícolas, a redução CRA pode ser acompanhada de decréscimo da fotossíntese, modificações de características fisiológicas e consequentemente, a produtividade vegetal (Bhagsari et al., 1976; Prisco, 1986, Dutra et al., 2011). O uso de tecnologias, com auxílio de novos sistemas sensores derivados do sensoriamento remoto, estima as necessidades hídricas culturas agrícolas e florestais em diferentes estádios de crescimento (Peñuelas \& Inoue, 1999; Moreira, 2001).

Neste contexto, o objetivo deste trabalho foi analisar o comportamento da assinatura espectral de 11 genótipos do gênero Eucalyptus spp.. Além de verificar o efeito da variação progressiva de perda do conteúdo relativo de água foliar no comportamento espectral, pelo método de desidratação, e identificar a região do espectro mais afetada pela variação do CRA.

\section{MATERIAL E MÉTODOS}

O estudo foi realizado no sítio experimental de número 36 (Piracicaba-SP), instalado em novembro de 2013, pertencente a rede do Programa Cooperativo de Tolerância de Eucalyptus spp. clonais aos Estresses Hídricos, Térmico e Biótico - TECHS (www.ipef.br/techs) do Instituto de Pesquisas e Estudos Florestais (Binkley et al., 2017). O sítio localiza-se na Escola Superior de Agricultura "Luiz de Queiroz", nas coordenadas geográficas $22^{\circ} 41^{\prime} 07^{\prime \prime} \mathrm{S}$ e $47^{\circ} 38^{\prime} 38^{\prime \prime}$ W e 
altitude de $550 \mathrm{~m}$. O solo local foi classificado como Nitossolo Vermelho distrófico com textura argilosa com drenagem moderada (Vidal-Torrado \& Sparovek, 1993). O clima da região é mesotérmico, tipo Cwa (Alvares et al., 2013), com verões quentes e chuvosos e invernos moderadamente frios e secos, com temperatura média anual de $19,1^{\circ} \mathrm{C}$ e a precipitação média anual de $1170 \mathrm{~mm}$.

Foram avaliados 11 genótipos comerciais de Eucalyptus spp. juntamente com Projeto TECHS (Binkley et al., 2017): a) genótipos tropicais, incluem E. grandis x E. camaldulensis, E. urophylla, E. urophylla $\times$ spp.; b) genótipos subtropicais, incluem E. benthamii, com E. dunnii, $E$. grandis, E. urophylla $\times$ E. grandis; c) genótipos plásticos, incluem E. grandis $\times E$. urophylla, $E$. urophylla, E. urophylla $\times$ E. globulus, e E. urophylla $\times$ E. tereticornis. Cada genótipo foi plantado em espaçamento $2,2 \mathrm{~m} \times 2,3 \mathrm{~m}$, portanto com uma área de $5,06 \mathrm{~m}^{2}$. planta ${ }^{-1}$, sendo 20 árvores úteis por parcela.

Para a amostragem foliar, foram selecionadas e marcadas quatro árvores de Eucalyptus spp. em cada parcela. Em seguida, foi feita uma separação visual da copa em superior, média e inferior (um terço para cada parte). Para cada árvore selecionada, foram coletadas quatro folhas, em quatro pontos opostos, e em cada terço da copa. Este procedimento foi padronizado para todos os genótipos. A folha selecionada foi sempre a segunda após a gema axilar do ramo com um aspecto visualmente saudável, plenamente desenvolvida e com boas condições fisiológicas, como critério de inclusão na amostragem.

A coleta das folhas ocorreu nos dias 9, 11 e 12 de dezembro de 2015, entre 7:00 e 9:00 horas. Após a coleta do material, as folhas foram identificadas, embaladas em sacos plásticos transparentes, e colocadas em caixa térmica com gelo (Summy et al., 2011).

O CRA é um parâmetro dado pela razão entre o teor de água no momento da coleta e o teor máximo de água (estado de turgor) para uma dada folha (Hunt Junior et al., 1987). Foi necessário determinar o peso fresco, o peso túrgido e peso seco da folha inteira, através da Equação 1 sugerida por Turner (1981) que utilizou originalmente discos foliares:

$\mathrm{CRA}=\left(\frac{\mathrm{PF}-\mathrm{PS}}{\mathrm{PT}-\mathrm{PS}}\right) * 100$

Em que PF (g) é o peso fresco, PS (g) é o peso seco após 72 horas na estufa a $70^{\circ} \mathrm{C}$ e PT (g) é o peso túrgido.

As folhas coletadas em campo foram levadas para o laboratório e pesadas imediatamente em uma balança analítica de precisão para a aquisição dos valores de massa úmida, a qual gerou o CRA para as folhas frescas ( $\left(C A_{\cup}\right)$. Após a pesagem, foi realizada a leitura espectral. Em seguida, as folhas foram imergidas em água destilada por 24 horas e pesadas para a obtenção da massa túrgida, e posteriormente foi realizada uma leitura espectral foliar correspondente ao máximo teor de água.

A leitura radiométrica do material vegetativo foi realizada com o espectroradiômetro FieldSpec ${ }^{\circledR}$ 3. O aparelho opera na faixa de $350 \mathrm{~nm}$ a $2500 \mathrm{~nm}$ do espectro eletromagnético, com uma resolução espectral de $10 \mathrm{~nm}$ (Analytical Spectral Device Inc., Boulder, Colorado, EUA). A radiância mensurada pelo sensor é convertida em reflectância através da calibração do sensor com a placa branca Spectralon presente no Contact probe acoplado ao Leaf clip (Oumar \& Mutanga, 2010).

O CRA foi obtido pela secagem sucessiva das amostras de folhas em uma estufa microbiológica a $40^{\circ} \mathrm{C}$, com tempo de secagem limitado a 30 minutos. Depois as folhas foram pesadas para obtenção de um novo valor de massa, que substituiu o valor de peso fresco na equação. Após cada pesagem, era obtida a resposta espectral correspondente. Esse processo foi repetido sete vezes (S1, S2, S3, S4, S5, S6 e S7) até que os valores de CRA Tempo $_{\text {ficarem }}$ constantes. Os pesos intermediários (PFT), dado em gramas, eram substituídos nos valores de Peso Fresco, assim como descrito por Fabre et al. (2011) (Equação 2): 
$\mathrm{CRA}_{\mathrm{Tempo}}=\left(\frac{\mathrm{PFT}-\mathrm{PF}}{\mathrm{PT}-\mathrm{PS}}\right) * 100$

No final das secagens intermediárias, as amostras de folhas foram colocadas em uma estufa a $70^{\circ} \mathrm{C}$ por 72 horas, para obtenção do peso seco.

Realizou-se uma análise de variância (ANOVA) para verificar se as amostras de CRA eram independentes quando estavam no tempo úmido. Outra ANOVA foi feita para verificar diferença estatística entre os CRA por tempo de secagem. Foi feito um teste de Tukey para comparação de médias. A análise estatística foi feita no software estatístico R (R Development Core Team, 2014).

Para melhor entendimento do efeito da variação do CRA na resposta espectral, foi calculada a diferença entre o fator de reflectância $\left(\mathrm{R}_{\text {Túrgida }}\right.$ menos $\left.\mathrm{R}_{\text {Tempo }}\right)$ de cada tempo de secagem e o fator de reflectância das folhas, que se encontravam no estado túrgido. Um gráfico de análise de sensibilidade espectral foi gerado pela divisão das curvas de diferença de reflectância com fator de reflectância no tempo túrgido (Carter, 1991).

Uma ACP das curvas espectrais foi realizada no software Parles 3.1 (Viscarra Rossel, 2008), a fim de reduzir a dimensionalidade dos dados espectrais originais e gerar uma tendência de variação dos dados em função da redução de água nas folhas e do gênero Eucalyptus spp.. Em seguida, no R, aplicou-se um teste de Tukey $(p<0,05)$ nesses comprimentos de onda para comparação entre as médias.

\section{RESULTADOS E DISCUSSÃO}

O fator de reflectância dos 11 genótipos estudados tiveram o mesmo comportamento (Figura 1), com bandas de absorção na região de 950nm - 970 nm, 1150 nm - 1260 nm, $1450 \mathrm{~nm}$ e $1950 \mathrm{~nm}$, que correspondem as bandas de absorção da água (Ceccato et al., 2002; Ranjan et al., 2015).

O E. benthamii se diferenciou espectralmente dos outros, pois teve maior intensidade no fator de reflectância. Essa diferença chegou a 7,3\% na região do azul (próximo a 445 nm) e 6,9\% na região do vermelho (próximo a $645 \mathrm{~nm}$ ). Em contraposição, a região do infravermelho próximo (720 nm - $1100 \mathrm{~nm}$ ) e infravermelho médio (1100 nm - $2500 \mathrm{~nm}$ ) alcançaram menor intensidade no fator de reflectância (Figura 1) (Jacquemoud et al., 1996).

Dentro do gênero Eucalyptus spp. existem diferentes espessuras de parece celular, parênquimas paliçádico e lacunoso, além dos elementos vasculares que propiciam árvores com diferentes tamanhos, densidade e qualidade da madeira (Foelkel et al., 1975; Tomazello Filho, 1981). Os genótipos deste estudo pertencem a diferentes grupos climáticos, com clones tropicais, subtropicais e plásticos (Binkley et al., 2017) e esta diferença não afeta CRA úmido, que é semelhante para todos os genótipos. O teste ANOVA mostrou que não existe diferença estatística a nível de $1 \%$ entre os $\mathrm{CRA}_{U}(\mathrm{~F}=0,4567$ e $\mathrm{p}=0,9)$. Apesar de o CRA não ter apresentado diferença estatística, O E. benthamii teve maior absorção de radiação eletromagnética na região do infravermelho. Portanto, a menor reflectância não foi relacionada com a quantidade de água na folha, mas provavelmente com a estrutura do mesófilo foliar menos lacunoso. Em uma célula vegetal mais jovem ocorre menor refração de água/ar nos espaços intercelulares e menor espalhamento de energia (Gates et al., 1965; Ponzoni et al., 2012).

O comportamento espectral de E. grandis e E. urophylla $\times$ E. grandis (Figura 1) revelou uma resposta semelhante, com um fator de reflectância próximo a $4 \%$ na banda do azul e de 4,2\% no vermelho. Silva et al. (2012) encontraram uma reflectância próxima à $5 \%$ na banda do vermelho ao estudar o comportamento espectral do E. grandis.

Ao analisar o infravermelho médio, encontrou-se maior diferença entre $E$. benthamii e $E$. dunnii, que chegou a 4,6\% (Figura 1). Resultado semelhante foi identificado no fator de reflectância entre E. benthamii e E. urophylla x E tereticornis (Figura 1) de 4\% no comprimento de onda $1400 \mathrm{~nm}$. Essa diferença no fator de reflectância, provavelmente, está relacionada à 
estrutura celular dos diferentes genótipos e não à quantidade de água representada pelo $\mathrm{CRA}_{\cup}$, pois os genótipos apresentaram um CRA próximo a $90 \%$.

Ao observar os genótipos por grupos climáticos, notou-se que os plásticos apresentaram maior variação média do CRA, indicando menor capacidade de armazenamento da água em suas células, seguido pelo subgrupo de genótipos subtropicais e tropicais, respectivamente.

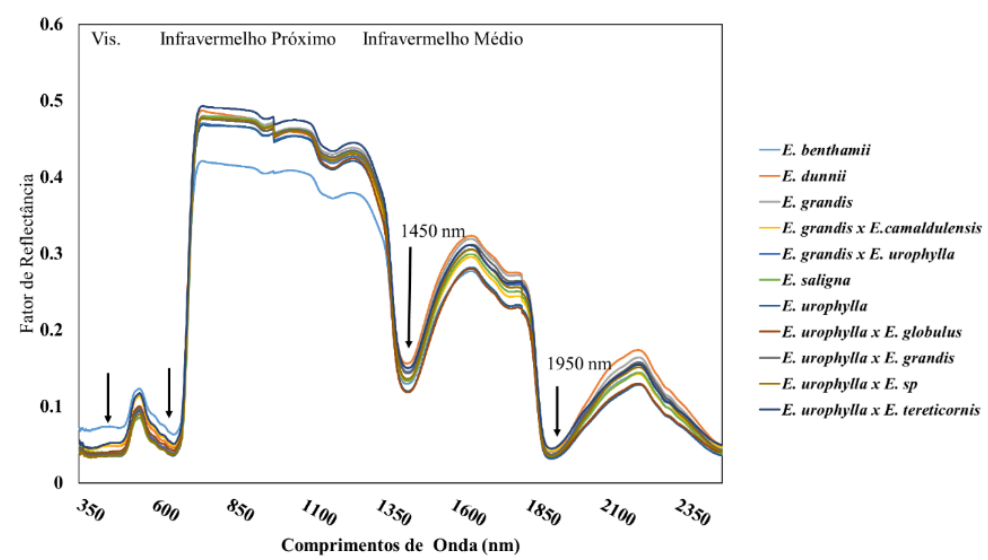

Figura 1. Comportamento espectral médio dos diferentes genótipos de Eucalyptus spp., após serem coletadas em campo com conteúdo relativo de água para folhas frescas (CRA $)$ ).

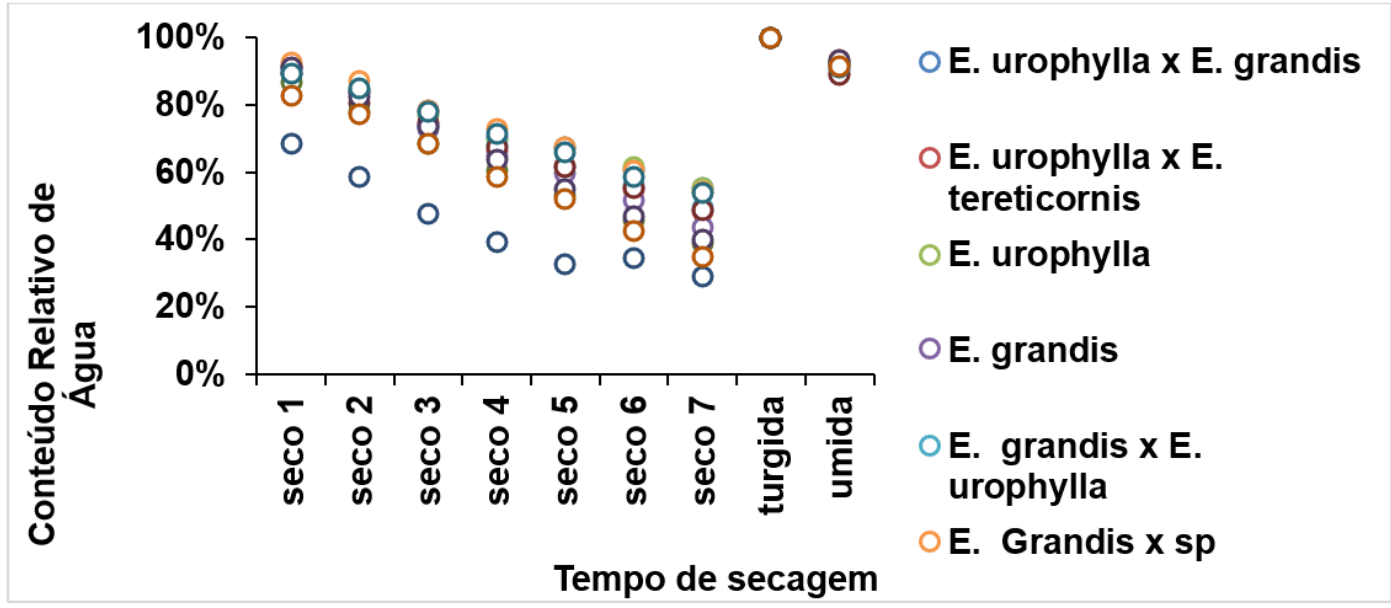

Figura 2. Representação gráfica dos valores médios do CRA dos diferentes genótipos de Eucalyptus spp., em diferentes tempos de secagem (S1, S2, S3, S4, S5, S6, S7), tempo túrgido (T) e úmido (U).

Cao et al. (2015) analisaram a desidratação de folhas de cinco genótipos diferentes com base no parâmetro CRA, e notaram que todos possuíam um valor similar de CRA inicial, assim não houve diferença estatística entre o CRA dos genótipos.

Dentre todos os genótipos observados, E. grandis $\times$ E. camaldulensis apresentou menor resistência a perda de água, com uma variação descendente de 70,97\% no CRA, do momento em que a folha se encontrava no estado túrgido ( $T$ ) até a última vez em que a mesma passou pela estufa (S7) (Figura 2). Durante a primeira secagem das folhas deste genótipo, foi observada uma perda expressiva de água. Após esse evento, a perda de água manteve-se constante e igual a $11 \%$ até o tempo $\$ 4$, posteriormente apresentou valores menores de perda de água. No tempo 56 , apresentou um $\mathrm{CRA}_{56}$ maior que o $\mathrm{CRA}_{55}$, provavelmente pela absorção de água do ambiente.

$O$ E. benthamii foi o segundo genótipo que perdeu mais água ao ter a primeira passagem pela estufa (S1) (Figura 2). As respostas fisiológicas de seis genótipos de Eucalyptus spp., inclusive o $E$. benthami, quando submetidos ao déficit hídrico, apresentaram um CRA semelhante (entre $80 \%$ - 90\%), antes da escassez de água (Merchant et al., 2007). Para os 
autores, esses genótipos tiveram uma alta tolerância ao stress, pois conseguiram manter suas funções celulares, mesmo com a queda da água na folha (Merchant et al., 2007).

Tanto o E. saligna quanto o E. urophylla $\times$ E. spp., que pertencem ao subgrupo dos genótipos plásticos, também apresentaram uma alta variação entre CRA nos tempos de secagem. Esses genótipos apresentaram um CRA inferior a 40\% no último tempo de secagem (S7) (Figura 2), porém E. saligna perdeu 13,58\% de água na primeira passagem pela estufa (S1).

E. urophylla e E. urophylla $\times$ E. grandis apresentaram a menor diferença entre o $\mathrm{CRA}_{T} \mathrm{e}$ $\mathrm{CRA}_{57}$. Essa desigualdade foi de $45 \%$, partindo de um CRA $\mathrm{A}_{\cup}$ semelhante, sendo o primeiro com um CRA de $90 \%$ e o segundo $92 \%$. Esses genótipos apresentaram um comportamento de perda de água similar nos tempos S7 (Figura 2). Fernandes et al. (2014), ao estudar a resposta fisiológica do $E$. urophylla $\times$ E. camaldulensis e E. urophylla $\times$ E. grandis e $E$. urophylla cultivados em casa de vegetação sob deficiência hídrica, encontraram que o segundo genótipo apresentou o CRA significativamente inferior aos outros. Neste estudo, E. urophylla $\times$ E. grandis apresentou um CRA superior quando submetido ao mesmo processo de secagem.

Em cada tempo na estufa, as folhas perderam água de forma heterogênea (Figura 2) e foi possível identificar uma variação na curva média do fator de reflectância foliar, principalmente na região do infravermelho, referente ao aumento com a perda de água entre1300 nm - 2500 $\mathrm{nm}$. Diversos estudos obtiveram resultados similares de aumento na reflectância com a queda do conteúdo de água na folha entre 350 nm - 700 nm e 1300 nm - 2500 nm (Carter, 1991; Cao et al., 2015).

A análise de variância (ANOVA) não mostrou distinção de genótipo na média de CRA por tempo de secagem $(F=108,662$ e $p<0,001)$. Portanto, houve diferença estatística entre as médias do CRA por tempo de secagem.

A média do CRA foi estatisticamente diferente no tempo túrgido no teste de Tukey (Tabela 1), enquanto que os outros tempos foram estatisticamente semelhantes ao tempo posterior ou anterior. Assim, a metodologia empregada em relação ao tempo e temperatura foi eficiente.

Tabela 1. Teste de Tukey para as médias de conteúdo relativo de água em diferentes tempos de secagem para 11 genótipos de Eucalyptus spp. estudados.

\begin{tabular}{ccccccccc}
\hline Úmida & Túrgida & Seco 1 & Seco 2 & Seco 3 & Seco 4 & Seco 5 & Seco 6 & Seco 7 \\
\hline $0,913^{b}$ & $1^{\mathrm{a}}$ & $0,872^{\mathrm{bc}}$ & $0,801^{\mathrm{c}}$ & $0,713^{\mathrm{d}}$ & $0,634^{\mathrm{e}}$ & $0,573^{\mathrm{ef}}$ & $0,513^{\mathrm{fg}}$ & $0,449^{\mathrm{g}}$
\end{tabular}

*médias seguidas pela mesma letra não diferem estatisticamente pelo teste de comparação de médias de Tukey a 95\% de probabilidade de confiança.

Para a análise descritiva da resposta espectral das folhas de Eucalyptus spp. submetidas a perda progressiva do CRA, foram selecionados dois genótipos, que mostraram resultados mais significativos para diferença de reflectância (Figura 3B, Figura 4B).

No $E$. grandis $\times$ E. camaldulensis foi observado uma resposta espectral característica de vegetação sadia, independentemente da quantidade de água encontrada na folha (Figura 3). $\mathrm{Na}$ região do visível, o genótipo apresentou o mesmo comportamento para o $\mathrm{CRA}_{T}$ e $\mathrm{CRA}_{U}$ com um pico de reflectância de $12 \%$ próximo aos $550 \mathrm{~nm}$, que está relacionado à quantidade de clorofila na folha (Schepers et al., 1996). Devido à perda de água foliar, essa região apresentou alterações que podem estar relacionadas com o fechamento dos estômatos (Souza et al., 2004) e com o rompimento da clorofila (Sun et al., 2008).

A dessecação das folhas teve alta influência sobre a resposta espectral, mas essa alteração pode estar relacionada a outros fatores que não somente a água. Apesar de pertencerem ao mesmo gênero, os genótipos tiveram uma perda de água de forma desuniforme e suas respostas espectrais seguiram a mesma tendência para perda da água. Foley et al. (2006) verificaram que o processo de perda da água variou por genótipo devido a organização e estrutura das células diferentes do mesófilo foliar.

O aumento da reflectância com a dessecação das folhas também foi encontrado nos comprimentos de onda de $3000 \mathrm{~nm}$ à $15000 \mathrm{~nm}$ no processo de secagem em folhas de 
cerejeira (Fabre et al., 2011). Os autores verificaram que a reflectância nessa região aumentou com a queda do conteúdo de água nas folhas.
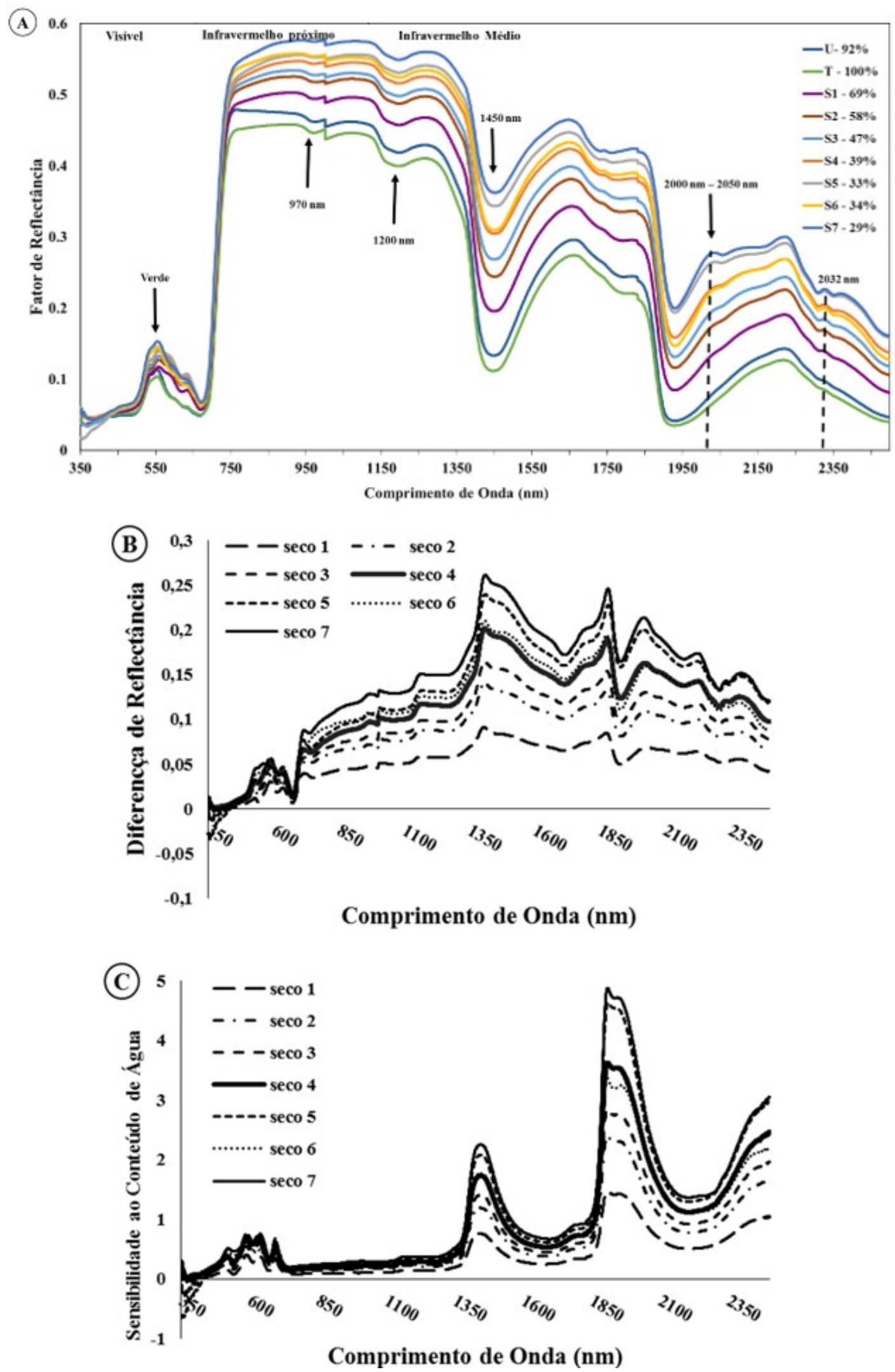

Figura 3. A- Comportamento espectral médio de diferentes conteúdos relativos de água em folhas de E. grandis $\times$ E. camaldulensis, B- Diferença entre a reflectância pelos comprimentos de onda, CSensibilidade da Reflectância para o conteúdo relativo de água. 
No $C R A_{T}$ (Figura $3 \mathrm{~A}$ ), as folhas se encontraram no estado túrgido quando a curva espectral exibiu menor intensidade no fator de reflectância para regiões do infravermelho próximo e médio. Ao comparar com a intensidade da reflectância de menores conteúdos de água na folha, a diferença no fator reflectância começou a se destacar a partir de $770 \mathrm{~nm}$. Gates et al. (1965) afirmaram que a reflectância de folhas no infravermelho próximo (IVP) foi o resultado da interação da energia incidente com a estrutura do mesófilo, e que a disponibilidade de água nessas células pode causar alteração da relação água/ar do mesófilo.

Ao analisar a resposta espectral em 970 nm, que corresponde à banda de absorção de energia devido a água, observou-se que a "calha" de absorção de energia começou a aumentar a reflectância no $\mathrm{CRA}_{53}(\mathrm{CRA}=47 \%)$. Peñuelas \& Inoue (1999) também perceberam que a absorção de energia diminuía com a dessecação na região de $970 \mathrm{~nm}$ para plantas de estrutura foliar diferentes, como o amendoim e o trigo.

A absorção de energia devido a água ocorre em torno de 980 a $1200 \mathrm{~nm}$, após o processo de fotólise (Gao, 1996; Meneses et al. 2019). Na Figura 3A, apesar da queda no CRA, essa região continuou sendo de absorção de energia. Mirzaie et al. (2014) e Zhang et al. (2015) corroboram com esse resultado, pois a redução da água na folha teve um aumento na reflectância dos comprimentos de onda de $1450 \mathrm{~nm}$ e $1950 \mathrm{~nm}$.

Entre os tempos $\mathrm{S} 5$ e $\mathrm{S} 6$ do E. grandis $\times$ E. camaldulensis, existiu uma variação ascendente do CRA com um acréscimo de 1\% (Figura 3A). Resultado coerente com a curva espectral, que teve um maior fator de reflectância no $\mathrm{CRA}_{55}$ que no $\mathrm{CRA}_{56}$, principalmente entre $1400 \mathrm{~nm}$ $1900 \mathrm{~nm}$. A curva S5 apresentou valores maiores que S6, revelando a sensibilidade da resposta espectral a essa pequena variação do CRA.

No $C_{R A}$, percebeu-se um aumento na intensidade da reflectância entre $2000 \mathrm{~nm}$ - 2050 nm (Figura 3A). A partir desse instante, a curva passou a apresentar um abaulamento, caracterizado por um aumento na reflectância, que se intensificou com a perda de água. Aproximadamente em $2320 \mathrm{~nm}$, identificou-se mais um pico de reflectância que começou a se destacar a partir do $\mathrm{CRA}_{52}$, que ficou mais evidente com a menor quantidade de água na folha. Dentre todos os genótipos de Eucalyptus spp. estudados, o genótipo apresentado na Figura $3 \mathrm{~A}$ foi o único que teve essa variação na assinatura espectral na região do infravermelho médio.

Na Figura 3B, a maior diferença foi encontrada no infravermelho, com uma pequena diferença apresentada na região do visível. Para todas as diferenças de reflectância, o valor máximo estava próximo a $1400 \mathrm{~nm}$ e $1900 \mathrm{~nm}$. A diferença da reflectância S7 (diferença entre o fator de reflectância do $\left(R A_{T}\right.$ e $\left(R A_{S 7}\right)$ não apresentou variação no começo do espectro, enquanto que essa variação teve uma amplitude máxima que chegou a $26 \%$ no $1400 \mathrm{~nm}$ e $22 \%$ no $1855 \mathrm{~nm}$. Uma diferença de reflectância negativa foi encontrada antes de $450 \mathrm{~nm}$ para o S2, S3 e S5, ou seja, a folha no momento túrgido tinha uma maior absorção de energia que nos outros tempos de secagem, provavelmente pela queda das atividades fotossintéticas (Araújo \& Deminicis, 2009).

De acordo com a Figura 2, o E. benthamii foi o segundo genótipo que apresentou maior variação no CRA da folha. No início da região do infravermelho médio (1100 nm até $1350 \mathrm{~nm}$ ) foram observados alguns tempos de secagem em que a reflectância da folha foi similar a reflectância do tempo anterior, como o $\mathrm{CRA}_{51}$ e o $\mathrm{CRA}_{52}$ (Figura 4). A maior diferença na reflectância aconteceu próximo de $1250 \mathrm{~nm}$ e foi de $9 \%$ entre o $\mathrm{CRA}_{55}$ e CRA $\mathrm{C}_{56}$. Nesse mesmo intervalo, percebeu-se que a diferença entre o CRA do tempo S6 e S7 foi de 6\% (Figura 2), porém a variação na reflectância foi imperceptível. A diferença de reflectância do $C R A_{T}$ e $C R A_{S 1}$ também foi negativo entre $950 \mathrm{~nm}-1350 \mathrm{~nm}$ (Figura 4B).

O E. benthamii se diferenciou dos outros genótipos devido a menor intensidade de reflectância na região do infravermelho. Ao verificar a sensibilidade do espectro em função do conteúdo de água (Figura 4C), a folha apresentou $\mathrm{CRA}_{57}$ de $36 \%$. Portanto, este genótipo não alcançou o valor de 50\% de reflectância na região do infravermelho próximo (Figura 4A), enquanto que no $E$. grandis $\times$ E. camaldulensis esse valor chegou quase a $60 \%$. O E. benthamii apresentou maior absorção de radiação eletromagnética na região do infravermelho por 
causa da estrutura do mesófilo foliar menos lacunoso, acarretando em um menor espalhamento da energia.
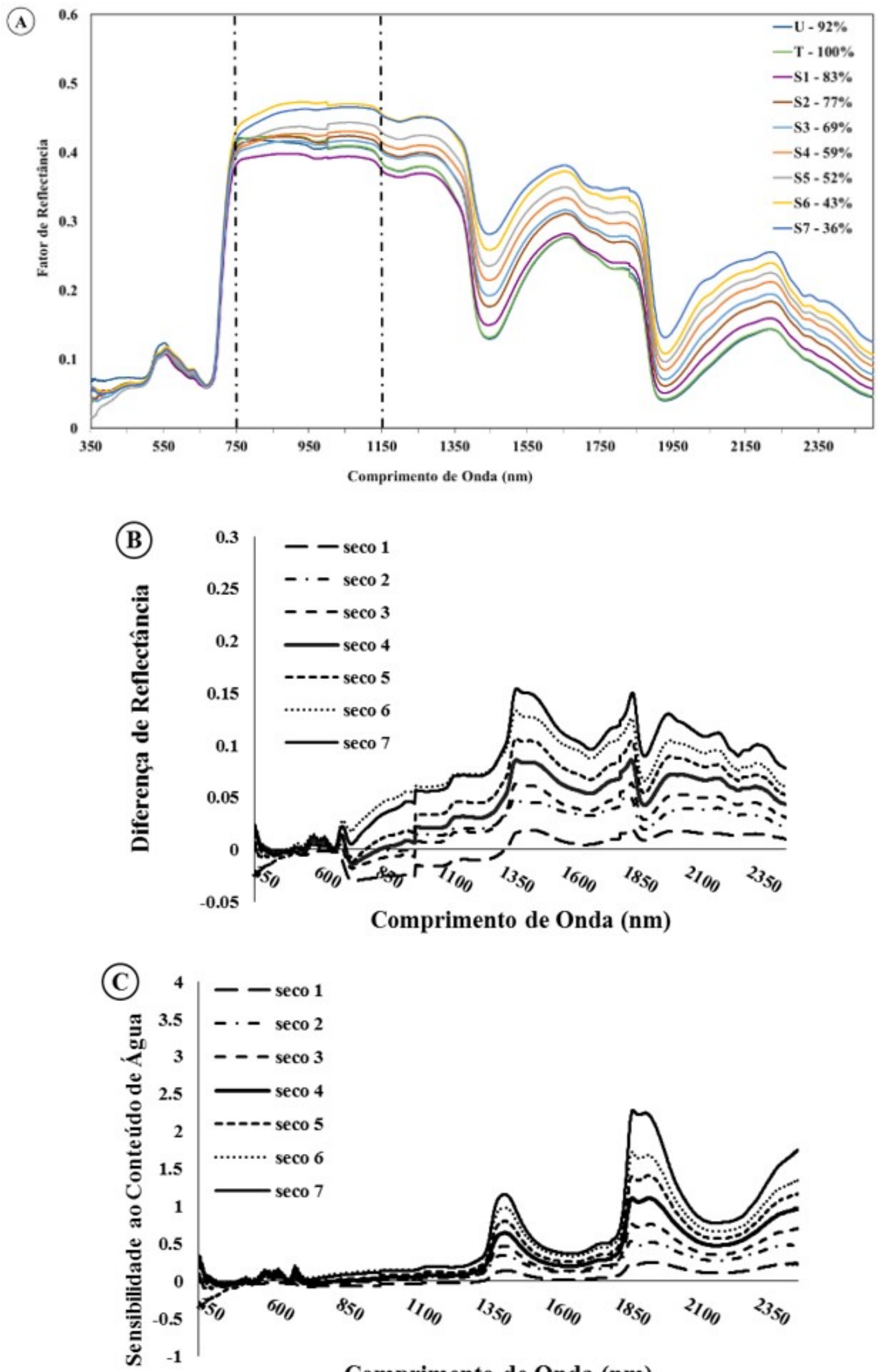

\section{Comprimento de Onda (nm)}

Figura 4. A-Comportamento espectral médio de diferentes conteúdos relativos de água em folhas de Eucalyptus benthamii, B- Diferença entre a reflectância pelos comprimentos de onda, C- Sensibilidade da Reflectância para o conteúdo relativo de água. 
Ao analisar os $\mathrm{CRA}_{56}$ e $\mathrm{CRA}_{57}$, entre $750 \mathrm{~nm}-1300 \mathrm{~nm}$, os genótipos pertencentes ao subgrupo plástico tiveram um aumento na reflectância significativo em relação a diminuição dos CRA's (Figura 4B). No E. benthamii, existiu uma inversão inesperada no fator de reflectância entre $750 \mathrm{~nm}$ e $1150 \mathrm{~nm}$ com queda de $7 \%$ entre $\mathrm{CRA}_{56}$ e CRA $\mathrm{C}_{57}$, que gerou uma diminuição na reflectância (Figura 4). Assim como, Fagus crenata teve uma resposta espectral inesperada entre os comprimentos de onda $750 \mathrm{~nm}$ e $1100 \mathrm{~nm}$, apesar de ter passado por uma variação de $48 \%$ para $16 \%$ no CRA, por causa da diferença de genótipo (Cao et al., 2015).

Na ACP das respostas espectrais médias por tempo de secagem existiu diferença do comportamento espectral pelo tempo de secagem (Figura 5A), assim como na análise descritiva. Os dois primeiros componentes principais (CP) explicaram 99,96\% da variância dos dados originais, sendo que a CP1 explicou 99,48\% e a CP2 explicou 0,47\% (Figura 5A). Os demais CP's tiveram uma baixa correlação com a variância dos dados e não foram incorporados na análise. A ferramenta multivariada possibilitou classificar os tempos de secagem em dois "scores" (CP1 e CP2). O "loading" do CP1 (Figura 5C) apresentou semelhança com o comportamento espectral da amostra e possuiu covariância positiva com todo o espectro, resultando em CP1 positivo. Enquanto, o "loading" da CP2 (Figura 5C) possuiu, visualmente, uma covariância positiva nas faixas do violeta e azul e entre $711 \mathrm{~nm}$ e $1346 \mathrm{~nm}$, e uma covariância negativa entre $1347 \mathrm{~nm}$ e $2500 \mathrm{~nm}$, sendo que em $1450 \mathrm{~nm}, 1887 \mathrm{~nm}$ e $2101 \mathrm{~nm}$ apresentaram a mais forte covariância.

Analisando-se a CP1, um grupo de novas variáveis se separou do restante das variáveis de forma distinta (Figura 5B). Assim, o E. benthamii se separou dos outros genótipos em relação à estrutura da vegetação, já que a CP1 teve uma alta influência da região entre $720 \mathrm{~nm}-1380 \mathrm{~nm}$ (Ponzoni et al., 2012), enquanto a CP2 apresentou maior distinção entre CRA's nos comprimentos de onda próximos de 1450 nm, $1950 \mathrm{~nm}$ e 2200 nm (Ranjan et al., 2015), bandas relacionada a absorção de água.

Quando as folhas diminuem o CRA, existiu uma variação ascendente tanto na CP1 quanto na CP2 (Figura 5A). A CP1 teve uma alta influência da região entre 720 nm e 1380 nm (Figura 5C), apesar de existir uma diferenciação entre os tempos de secagem na CP1. A CP2 apresentou maior distinção entre os CRA's, pois os tempos S2 e S3 na CP1 tiveram uma posição gráfica semelhante e o mesmo resultado foi obtido nos tempos de S6 e S7.

Foi evidente a variação ascendente e inversa da CP2 em função da água (Figura 5A), isto é, quanto menor o CRA das folhas, maior o valor na CP2. As variáveis de maior influência sobre a CP2 foram os comprimentos de onda próximos de 1450 nm, 1950 nm e 2200 nm, que são bandas relacionadas com a absorção de água (Ranjan et al., 2015).

A CP2 foi fortemente influenciada pelos comprimentos de onda $1450 \mathrm{~nm}, 1887 \mathrm{~nm}$ e $2101 \mathrm{~nm}$ (Figura 5C), portanto houve a variação da reflectância em função da secagem dessas bandas (Figura 6) e essa variação foi ascendente em relação a perda da água na folha.

O teste de Tukey foi realizado para verificar se existia diferença estatística entre os tempos de secagem dos picos espectrais de todos os genótipos de Eucalyptus spp., representados na CP2. Houve semelhança estatística com o fator de reflectância do tempo posterior ou anterior, para os três comprimentos de onda (Tabela 2). Em $1450 \mathrm{~nm}$, o fator de reflectância no momento túrgido passou a ter diferença estatística, após a terceira passagem pela estufa, este mesmo comportamento foi encontrado em $1887 \mathrm{~nm}$. Enquanto, a média do fator de reflectância das folhas no estado túrgido para $2101 \mathrm{~nm}$ apresentou diferença estatística com a média do fator de reflectância dos outros tempos de secagem, após a segunda passagem das folhas na estufa.

O conteúdo de água na folha influencia a reflectância entre 1300 a 2500 nm (Cao et al., 2015). Durante curtos períodos de desidratação, a reflectância aumentou à medida que diminui o teor de água foliar, sendo o intervalo mais adequado entre 1300 e $2000 \mathrm{~nm}$. Entretanto, podem existir níveis de absorção entre 2000 e 2500 nm, acarretando incertezas sobre recuperação de água das folhas desidratadas (Cao et al., 2015). 

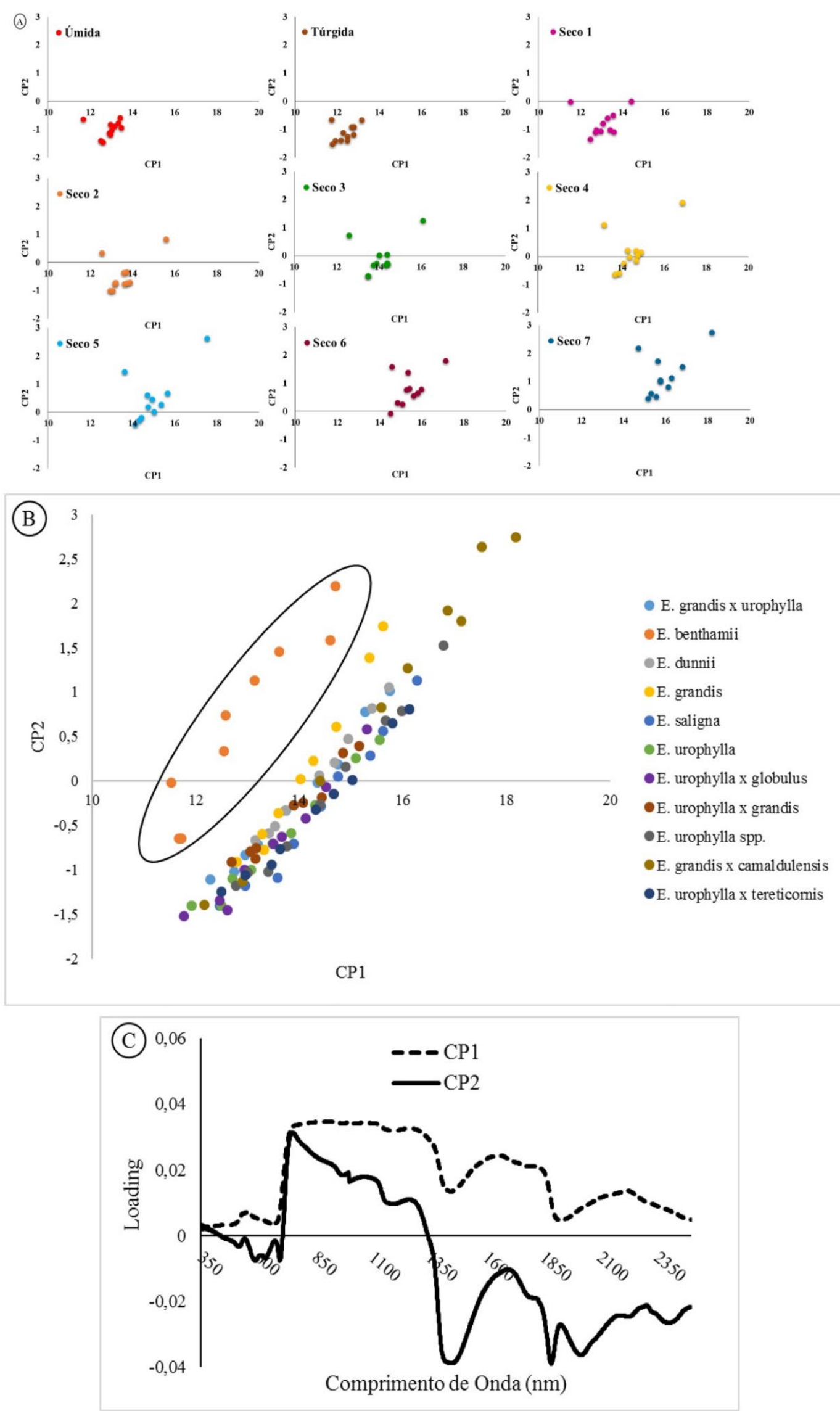

Figura 5. (A) Gráfico de Componentes Principais em função dos tempos de secagem, (B) Gráfico de Componentes Principais em função dos 11 genótipos estudados, (C) Gráfico de loadings das Componentes Principais dos dois primeiros scores. 


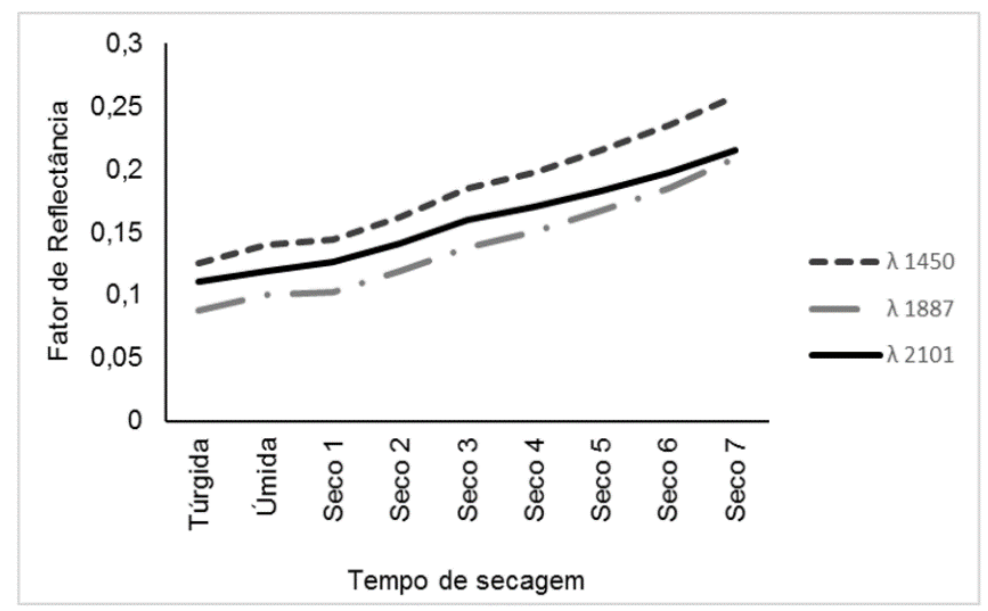

Figura 6. Variação do fator de reflectância (1450 nm, $1887 \mathrm{~nm}$ e $2101 \mathrm{~nm}$ ) em função do tempo de secagem para 11 genótipos de Eucalyptus spp. estudados.

Tabela 2. Teste de Tukey para o fator de reflectância das bandas ( $\lambda$ ) 1450 nm, $1887 \mathrm{~nm}$ e $2101 \mathrm{~nm}$ pelos tempos de secagem.

\begin{tabular}{ccccccc}
\hline & $\boldsymbol{\lambda 1 4 5 0}$ & \multicolumn{7}{c}{$\boldsymbol{\lambda \mathbf { 1 8 8 7 }}$} & $\boldsymbol{\lambda 2 1 0 1}$ & \\
\hline Túrgida & 0,12 & $\mathrm{f}$ & 0,08 & $\mathrm{~F}$ & $\mathbf{0 , 1 0}$ & $\mathrm{f}$ \\
Úmida & 0,131 & $\mathrm{f}$ & 0,09 & Ef & 0,11 & ef \\
S 1 & 0,14 & ef & 0,10 & Ef & 0,12 & ef \\
S 2 & 0,16 & def & 0,12 & def & 0,14 & de \\
S 3 & 0,18 & cde & 0,13 & cde & 0,15 & cd \\
S 4 & 0,20 & bcd & 0,15 & bcd & 0,17 & bcd \\
S 5 & 0,22 & bc & 0,17 & Bc & 0,18 & bc \\
S 6 & 0,24 & ab & 0,19 & Ab & 0,20 & ab \\
S 7 & 0,26 & a & 0,21 & A & 0,21 & a \\
\hline
\end{tabular}

*médias seguidas pela mesma letra não diferem estatisticamente pelo teste de comparação de médias de Tukey a 95\% de probabilidade de confiança.

\section{CONCLUSÃO}

A resposta espectral dos diferentes genótipos de Eucalyptus spp. apresenta um comportamento característico de uma vegetação sadia, sem modificação na resposta devido à deficiência hídrica. O E. benthamii foi uma exceção por apresentar um menor fator de reflectância no infravermelho próximo e médio.

É possível afirmar que a variação do conteúdo relativo de água tem influência inversa na resposta espectral em folha de Eucalyptus spp., pois quanto menor conteúdo de água na folha, maior o fator de reflectância, principalmente na região do infravermelho médio.

\section{REFERÊNCIAS}

Alvares, A. C., Stape, J. L., Sentelhas, P. C., Gonçalves, J. L. M., \& Sparovek, G. (2013). Koppen's climate classification map for Brazil. Meteorologische Zeitschrift, 22(6), 711-728.

http://dx.doi.org/10.1127/0941-2948/2013/0507.

Araújo, S. A. C., \& Deminicis, B. B. (2009). Fotoinibição da Fotossíntese. Revista Brasileira de Biociências, 7(4), 463-472.

Arndt, S. K., Irawan, A., \& Sanders, G. (2015). Apoplastic water fraction and rehydration techniques introduce significant errors in measurements of relative water content and osmotic potential in plant leaves. International Journal of Plant Biology, 155, 355-368. 
Bhagsari, A. S., Brown, R. H., \& Schepers, J. S. (1976). Effect of moisture stress on photosynthesis and some related physiological characteristics in peanut. Crop Science, 16(5), 712-715. http://dx.doi.org/10.2135/cropsci1976.0011183X001600050029x.

Binkley, D., Campoe, O. C., Alvares, C., Carneiro, R. L., Cegatta, Í., \& Stape, J. L. (2017). The interactions of climate, spacing and genetics on clonal Eucalyptus plantations across Brazil and Uruguay. Forest Ecology and Management, 405, 271-283. http://dx.doi.org/10.1016/j.foreco.2017.09.050.

Campoe, O. C., Munhoz, J. S. B., Alvares, C. A., Carneiro, R. L., Mattos, E. M., Ferez, A. P. C., \& Stape, J. L. (2016). Meteorological seasonality affecting individual tree growth in forest plantations in Brazil. Forest Ecology and Management, 380, 149-160. http://dx.doi.org/10.1016/j.foreco.2016.08.048.

Cao, Z., Wang, Q., \& Zheng, C. (2015). Best hyperspectral indices for tracing leaf water status as determined from leaf dehydration experiments. Ecological Indicators, 54, 96-107. http://dx.doi.org/10.1016/j.ecolind.2015.02.027.

Carter, G. A. (1991). Primary and secondary effects of water content on the spectral reflectance of leaves. American Journal of Botany, 78(7), 916-924. http://dx.doi.org/10.1002/j.15372197.1991.tb14495.x.

Ceccato, P., Gobron, N., Flasse, S., Pinty, B., \& Tarantola, S. (2002). Designing a spectral index to estimate vegetation water content from remote sensing data. Part 1 theoretical approach. Remote Sensing of Environment, 82(2-3), 188-197. http://dx.doi.org/10.1016/S0034-4257(02)00037-8.

Datt, B. (1999). Remote sensing of water content in Eucalyptus leaves. Australian Journal of Botany, 47(6), 909-923. http://dx.doi.org/10.1071/BT98042.

Dutra, A. D., Sampaio, A. H. R., Guimarães, M. J. M., Silva, R. O., Calbo, A. G., \& Coelho Filho, M. A. (2011). Relação entre conteúdo relativo de água e potencial de turgor obtido com wiltmeter em folhas de mamoeiro. In Anais do $5^{\circ}$ Simpósio do Papaya Brasileiro: Inovações e Sustentabilidade (pp. 1-4). Porto Seguro: Embrapa Madioca e Fruticultura.

Elli, E. F., Sentelhas, P. C., de Freitas, C. H., Carneiro, R. L., \& Alvares, C. A. (2019). Intercomparison of structural features and performance of Eucalyptus simulation models and their ensemble for yield estimations. Forest Ecology and Management, 450, 117493. http://dx.doi.org/10.1016/j.foreco.2019.117493.

Fabre, S., Lesaignoux, A., Olioso, A., \& Briottet, X. (2011). Influence of water content on spectral reflectance of leaves in the 3-15 $\mu \mathrm{m}$ domain. IEEE Geoscience and Remote Sensing Letters, 8(1), 143 147. http://dx.doi.org/10.1109/LGRS.2010.2053518.

Fernandes, E. T., Cairo, P. A. R., \& Novaes, A. B. (2014). Resposta fisiológica de clones de eucalipto cultivados em casa de vegetação sob deficiência hídrica. Ciência Rural, 45(1), 29-34. http://dx.doi.org/10.1590/0103-8478cr20120152.

Flores, T. B., Alvares, C. A., Souza, V. C., \& Stape, J. L. (2018). Eucalyptus in Brazil: climatic zoning and identification guide. Piracicaba: IPEF.

Foelkel, C. E. B., Barrichelo, L. E. G., \& Milanez, A. F. (1975). Estudo comparativo das madeiras de Eucalyptus saligna, E. paniculata, E. citriodora, E. maculata e E. tereticornis para produção de celulose sulfato. IPEF, (10), 17-37.

Foley, S., Rivard, B., Sanchez-Azofeifa, G. A., \& Calvo, J. (2006). Foliar spectral properties following leaf clipping and implications for handling techniques. Remote Sensing of Environment, 103(3), 265-275. http://dx.doi.org/10.1016/j.rse.2005.06.014.

Gao, B. (1996). NDWl: a normalized difference water index for remote sensing of vegetation liquid water from space. Remote Sensing of Environment, 58(3), 257-266. http://dx.doi.org/10.1016/S00344257(96)00067-3.

Gates, D. M., Keegan, H. J., Schleter, J. C., \& Weidner, V. R. (1965). Spectral properties of plants. Applied Optics, 4(1), 11-20. http://dx.doi.org/10.1364/AO.4.000011.

Hunt Junior, E. R., Rock, B. N., \& Nobel, P. S. (1987). Measurement of leaf relative water content by infrared reflectance. Remote Sensing of Environment, 22(3), 429-435. http://dx.doi.org/10.1016/00344257(87)90094-0.

Jacquemoud, S., Ustin, S., Verdebout, J., Schmuck, G., Andreoli, G., \& Hosgood, B. (1996). Estimating leaf biochemistry using the PROSPECT leaf optical properties model. Remote Sensing of Environment, 56(3), 194-202. http://dx.doi.org/10.1016/0034-4257(95)00238-3.

Jones, H. G. (1992). Plants and microclimate: a quantitative approach to environmental plant physiology (86 p.). Cambridge: Cambridge University Press. 
Meneses, P. R., Almeida, T., \& Baptista, G. M. M. (2019). Reflectância dos materiais terrestres: análise e interpretação. São Paulo: Oficina de Textos.

Merchant, A., Callister, A., Arndt, S., Tausz, M., \& Adams, M. (2007). Contrasting physiological responses of six Eucalyptus species to water deficit. Annals of Botany, 100(7), 1507-1515. http://dx.doi.org/10.1093/aob/mcm234.

Mirzaie, M., Darvishzadeh, R., Shakiba, A., Matkan, A. A., Atzberger, C., \& Skidmore, A. (2014). Comparative analysis of different uni-and multi-variate methods for estimation of vegetation water content using hyper-spectral measurements. International Journal of Applied Earth Observation and Geoinformation, 26, 1-11. http://dx.doi.org/10.1016/j.jag.2013.04.004.

Moreira, M. A. (2001). Fundamentos do sensoriamento remoto e metodologia de aplicação. São José dos Campos: Instituto Nacional de Pesquisas Espaciais.

Oumar, Z., \& Mutanga, O. (2010). Predicting plant water content in Eucalyptus grandis forest stands in KwaZulu-Natal, South Africa using field spectra resampled to the Sumbandila Satellite Sensor. International Journal of Applied Earth Observation and Geoinformation, 12(3), 158-164. http://dx.doi.org/10.1016/j.jag.2010.02.002.

Peñuelas, J., \& Inoue, Y. (1999). Reflectance indices indicative of changes in water and pigment contents of peanut and wheat leaves. Photosynthectica, 36(3), 355-360. http://dx.doi.org/10.1023/A:1007033503276.

Ponzoni, F. J., Shimabukuro, Y., \& Kuplich, T. M. (2012). Sensoriamento remoto no estudo da vegetação. São José dos Campos: Oficina de Texto.

Prisco, J. T. (1986). Possibilidades de exploração de lavouras xerófilas no semi-árido brasileiro. Pesquisa Agropecuária Brasileira, 21(4), 333-342.

R Development Core Team (2014). A Language and Environment for Statistical Computing. Vienna, Austria: R Foundation for Statistical Computing.

Ranjan, R., Sahoo, R. N., Chopra, U. K., Pramanik, M., Singh, A. K., \& Pradhan, S. (2015). Assessment of water status in wheat (Triticum aestivum L.) using ground based hyperspectral reflectance. Biological Sciences, 5(20), 1-12.

Schepers, J. S., Blackmer, M. T., Wilhelm, W., \& Resende, M. (1996). Transmittance and reflectance measurements of corn leaves from plants with different nitrogen and water supply. Journal of Plant Physiology, 148(5), 523-529. http://dx.doi.org/10.1016/S0176-1617(96)80071-X.

Silva, E. A., Marangon, G. P., Dessbesell, L., Morais, W. W., Lippert, D. B., \& Pereira, R. S. (2012). Caracterização espectral na reflectância de Eucalyptus gradis. Revista Floresta, 42(2), 285-292. http://dx.doi.org/10.5380/rf.v42i2.17587.

Souza, R. P., Machado, E. C., Silva, J. A. B., Lagôa, A. M. M. A., \& Silveira, J. A. G. (2004). Photosynthetic gas Exchange, chlorophyll fluorescence and some associated metabolic changes in cowpea (Vigna unguiculate) during water stress and recovery. Environmental and Experimental Botany, 51(1), 45-56. http://dx.doi.org/10.1016/S0098-8472(03)00059-5.

Summy, K. R., Lieman, J., Gandy, Y. P., Mamachen, A., Goolsby, J., \& Moran, P. J. (2011). Effects of leaf excision and sample storage methods on spectral reflectance by foliage of giant reed, Arundo donax. Yaredai Zhiwu Kexue, 63, 54-64.

Sun, P., Grignetti, A., Liu, S., Casacchia, R., Salvatori, R., Pietrini, F., Loreto, F., \& Centritto, M. (2008). Associated changes in physiological parameters and spectral reflectance indices in olive (Olea europea L.) leaves in response to different levels of water stress. International Journal of Remote Sensing, 29(6), 1725-1743. http://dx.doi.org/10.1080/01431160701373754.

Sun, P., Wahbi, S., Tsonev, T., Haworth, M., Liu, S., \& Centritto, M. (2014). On the use of leaf spectral indices to assess water status and photosynthetic limitations in Olea europaea L. during waterstress and recovery. PLoS One, 9(8), e105165. http://dx.doi.org/10.1371/journal.pone.0105165.

Tomazello Filho, M. (1981). Estrutura anatômica da madeira de oito espécies de eucalipto cultivadas no Brasil. IPEF, (29), 25-36. http://dx.doi.org/10.1007/BF02180062.

Turner, N. C. (1981). Techniques and experimental approaches for the measurement of plant water status. Plant and Soil, 58(1-3), 339-366. http://dx.doi.org/10.1007/BF02180062.

Vidal-Torrado, P., \& Sparovek, G. (1993). Mapa pedológico detalhado 1: 10000 do Campus Luiz de Queiroz. Piracicaba: ESALQ-USP.

Viscarra Rossel, R. A. (2008). ParLeS: software for chemometric analysis of spectroscopic data. Chemometrics and Intelligent Laboratory Systems, 90(1), 72-83. http://dx.doi.org/10.1016/j.chemolab.2007.06.006. 
Zhang, C., Pan, Z., Dong, H., He, F., \& Hu, X. (2015). Remote estimation of leaf water content using spectral index derived from hyperspectral data. In Proceedings of the 1st International Conference on Information Science and Eletronic Technology (pp. 20-23). Wuhan: Atlantis Press. http://dx.doi.org/10.2991/iset-15.2015.6.

Contribuição dos Autores: TFS: Análise Formal, Metodologia, Investigação; PRF: Conceituação, Obtenção de Financiamento, Administração do Projeto, Supervisão; CAA: Escrita - Primeira Redação, Escrita - Revisão e Edição; ESN: Escrita - Primeira Redação, Escrita - Revisão e Edição 